\title{
Posterior lumbar interbody fusion with bioabsorbable spacers and local autograft in a series of 27 patients
}

\author{
Daniel E. Couture, M.D., and Charles L. Branch Jr., M.D. \\ Wake Forest University Medical Center, Winston-Salem, North Carolina
}

\begin{abstract}
Object. The goal of this prospective study was to review a series of 27 patients who underwent bilateral posterior lumbar interbody fusion with instrumented pedicle fixation and two HYDROSORB (known generically as 70:30 poly[L-lactide-co-D,L-lactide]) rectangular cages packed with locally harvested autograft at a total of 48 levels, and to assess the safety and efficacy of this novel technique. This analysis, conducted at a mean of 26 months of follow up, is the first report of a long-term evaluation of this technique. Fusion rates and clinical outcomes are presented.

Methods. A prospective clinical and radiographic review of findings in 27 consecutive patients was performed. Fusion rates and clinical outcome were assessed at 6-month intervals up to the 32-month follow-up end point. Two patients with four corresponding fusion levels were lost to follow up.

Radiographic evidence of satisfactory fusion was achieved in $42(95.5 \%)$ of 44 levels fused. Satisfactory fusion at all levels was achieved in $23(92 \%)$ of 25 patients. Two patients required repeated operations for treatment of symptomatic pseudarthrosis during the study period. The likelihood of all levels attaining fusion in a given patient decreased as the number of levels treated increased, which is consistent with previously published studies. Nonetheless, fusion rates per treated level were similar for patients in whom one to three levels were treated. No significant surgical complication occurred.

Conclusions. Posterior lumbar interbody fusion in which the HYDROSORB bioabsorbable implant packed with locally harvested autograft and segmental internal fixation are used appears to be an interbody fusion alternative whose efficacy is comparable with previously reported procedures.
\end{abstract}

\section{KEY WORDS • degenerative disc disease • recurrent disc disease • posterior lumbar interbody fusion $\bullet$ bioabsorbable implants • pedicle screw}

Posterior lumbar interbody fusion is a therapeutic option for patients who suffer from pain and instability of the lumbar spine. The first account of the PLIF procedure was published by Jaslow ${ }^{12}$ in 1946; this author used autograft bone wedges from the iliac crest as implants. When bone graft was used as a stand-alone device, however, there was a significant incidence of collapse and pseudarthrosis. ${ }^{14}$ The techniques involved in the PLIF procedure have been refined and advanced since then with the addition of pedicle screw fixation to supplement lumbar fusion constructs as well as intervertebral cages or spacers developed to prevent the collapse and pseudarthrosis found in noninstrumented fusions. There have been many new developments in bone support prostheses, including resorbable polymer implants. One such polymer, HYDROSORB (Medtronic Sofamor Danek, Memphis, TN) has been evaluated as a promising material in spinal surgery. ${ }^{2}$

HYDROSORB is a polyhydroxy acid, a copolymer composed of a 70:30 ratio of poly(L-lactide) to poly(D,Llactide) that is characterized by a degradation time of 18 to 36 months. There is extensive experience with polyhydroxy acid implants in Europe. This material does not induce a significant inflammatory or allergic reaction and is

Abbreviations used in this paper: $\mathrm{CT}=$ computerized tomography; PLIF = posterior lumbar interbody fusion. biocompatible with the dura mater and neuronal cells (unpublished data). It is not well visualized on routine radiographic studies but can be seen clearly on CT scans and magnetic resonance images prior to resorption without causing image degradation. Its strength and degradation characteristics allow it to provide mechanical support to the anterior column, but it may reduce stress shielding of the developing interbody fusion by the gradual reduction in load-sharing capacity as the structural integrity of the device is lost.

There are no published studies documenting the longterm clinical outcome or fusion rates attained using bioabsorbable implants for PLIF. We present the first experience in a series of 27 patients whose a mean follow-up duration was 26 months, and we evaluate the safety and efficacy of this technique.

\section{CLINICAL MATERIAL AND METHODS}

\section{Patient Population}

Between May 2001 and August 2002, 27 patients underwent PLIF procedures in which rectangular HYDROSORB interbody spacers packed with locally harvested autograft bone were used. Of these procedures, 14 were single level fusions, six were two-level fusions, six were three-level fusions, and one was a four-level fusion; one 
patient in the single-level and one in the three-level group were lost to follow up. The patients' characteristics, which include sex, age, tobacco use, Worker's compensation, and previous surgery, are presented in Table 1.

All patients presented with a combination of mechanical low-back pain, neurogenic claudication, and a significant radicular component that was refractory to nonsurgical management. Radiographic indications for fusion included plain $\mathrm{x}$-ray films showing instability and magnetic resonance images demonstrating degenerative changes, central or foraminal stenosis, and disc collapse. Patients were offered the opportunity to participate in a standardized clinical assessment program (PhDx Systems, Albuquerque, NM), and 21 patients elected to do so.

All patients underwent PLIF procedures with implantation of HYDROSORB devices packed with "morcellized" autograft bone harvested from the elements of decompression, followed by pedicle screw fixation and bilateral transverse process fusions. All procedures were performed by the senior author (C.L.B.).

\section{Surgical Procedure}

A midline incision was made and dissection was extended along the spinous process. Muscle stripping was performed to the level of the medial transverse process bilaterally just over the facet capsules. A bilateral hemilaminectomy decompression was created at the appropriate levels. Once the nerve root was identified, careful dissection was performed for visualization of the disc along the superior border of the pedicle. The target disc spaces were incised bilaterally and distracted with a disc space distractor. Intradiscal soft-tissue removal and endplate preparation were performed bilaterally according to the Tangent technique. A HYDROSORB device packed with locally harvested autologous morcellized bone graft was tamped into the disc space on one side. The center of the disc space was filled with morcellized graft and another HYDROSORB device was inserted on the second side.

Pedicle screws were placed in the pedicles of the appropriate levels, with bicortical purchase at S-1. Rods or plates were fixed to the pedicle screws in anatomical positions. Bone graft was then inserted over the transverse process of the lateral facet joint and the wound was closed in layers. Patients were instructed to wear a lumbar corset for 3 months.

\section{Postoperative Evaluation}

Patients in this series were evaluated for a minimum of 17 months, based on radiographs and physical examina-

TABLE 1

Characteristics in 27 patients who underwent PLIF procedures*

\begin{tabular}{ll}
\hline \hline \multicolumn{1}{c}{ Feature } & Value \\
\hline no. of patients & 27 \\
mean age in yrs (range) & $58(40-79)$ \\
mean FU in mos (range) & $26.1(17-32)$ \\
percentage w/ & \\
male sex & 48.1 \\
tobacco use & 14.8 \\
Worker's comp & 7.4 \\
previous surgery & 40.1 \\
\hline
\end{tabular}

tions. We report the success of the fusion at individual levels as observed on plain radiographs and CT scans. Fusion was defined as trabecular bone bridging the interspace around or within the implant, with the absence of increased lucency around the implant. The assessment of fusion and stability based on plain radiographs and intermittent CT scanning was performed by the senior author and by radiologists at our institution. In this review, a patient with an incomplete fusion on CT scans who met the fusion criteria on plain radiographs and in whom there were no clinical or radiographic signs of a pseudarthrosis was assigned to the successful fusion group. Clinical outcomes were assessed prospectively based on results of patient examinations and data recordings before surgery, at surgery, and at 1-, 3-, and 6-month intervals after surgery. Evaluations at each interval included ratings of pain, function, medication usage, and patient satisfaction. More extensive details of the clinical outcomes, including Short Form-36 and other clinical assessment tools, will be published in a subsequent manuscript.

\section{RESULTS}

A summary of fusion rates and levels is found in Table 2. Overall, successful fusion was achieved according to radiographic images at $42(95.5 \%)$ of 44 levels. When analyzed by the number of levels fused per patient, all single-level fusions were successful (Fig. 1). In two-level fusions, all 12 levels (100\%) were successfully fused (Fig. 2). In three-level fusions, $14(93.3 \%)$ of 15 levels were successfully fused (Fig. 3). In the four-level fusion, three $(75 \%)$ of four levels were successfully fused. Fusion success, when analyzed according to the number of patients undergoing single- or multiple-level fusions, revealed the following results. Single- and two-level fusions remained the same at $100 \%$. In patients with three-level fusions, four $(80 \%)$ of five attained successful fusions at all levels.

TABLE 2

Rates of satisfactory fusion in 27 patients who underwent PLIF procedures

\begin{tabular}{|c|c|c|c|c|}
\hline Extent of Op & $\begin{array}{l}\text { No. W/ } \\
\text { Attempted } \\
\text { Fusion }\end{array}$ & $\begin{array}{c}\text { No. W/ } \\
\text { Fusion/ } \\
\text { Stable/ } \\
\text { Incomplete } \\
\text { Results }\end{array}$ & $\begin{array}{c}\text { No. W/ } \\
\text { Failed } \\
\text { Ops }\end{array}$ & $\begin{array}{l}\% \mathrm{~W} / \\
\text { Fusion }\end{array}$ \\
\hline \multicolumn{5}{|l|}{ 1-level fusion } \\
\hline individual levels & 13 & 13 & 0 & 100.0 \\
\hline patients w/ 1-level fusions & 13 & 13 & 0 & 100.0 \\
\hline \multicolumn{5}{|l|}{ 2-level fusion } \\
\hline individual levels & 12 & 12 & 0 & 100.0 \\
\hline patients w/ 2-level fusions & 6 & 6 & 0 & 100.0 \\
\hline \multicolumn{5}{|l|}{ 3-level fusion } \\
\hline individual levels & 15 & 14 & 1 & 93.3 \\
\hline patients w/ 3-level fusions & 5 & 4 & 1 & 80.0 \\
\hline \multicolumn{5}{|l|}{ 4-level fusion } \\
\hline individual levels & 4 & 3 & 1 & 75.0 \\
\hline patients w/ 4-level fusions & 1 & 0 & 1 & 0.0 \\
\hline \multicolumn{5}{|l|}{ total levels } \\
\hline individual levels & 44 & 42 & 2 & 95.5 \\
\hline no. of patients & 25 & 23 & 2 & 92.0 \\
\hline
\end{tabular}



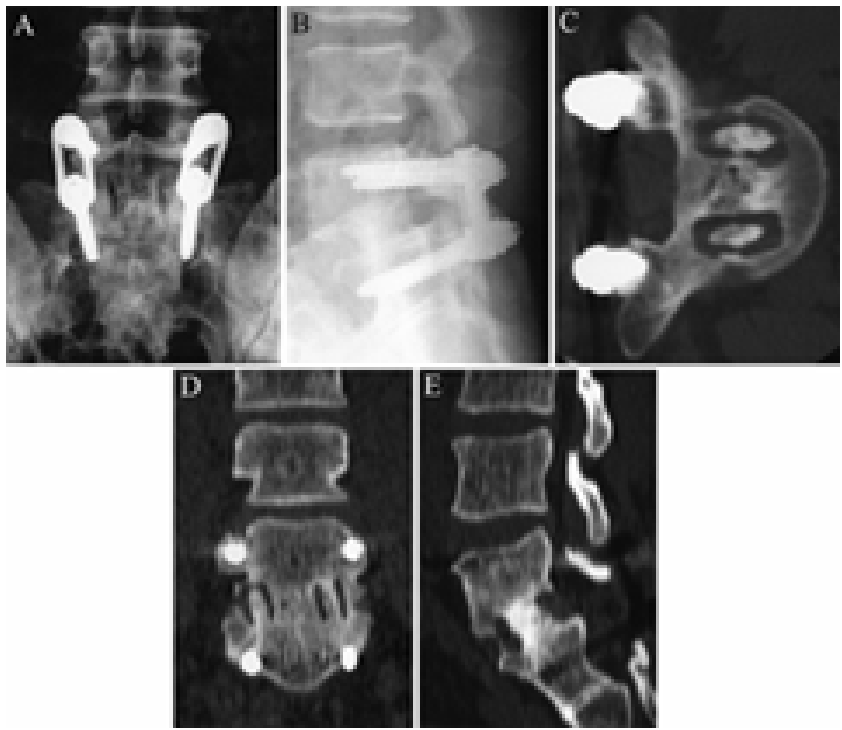

Fig. 1. Postoperative studies demonstrating single-level fusion at L5-S1 at 24-month follow-up review. A: An anteroposterior (AP) radiograph obtained 24 months after L5-S1 fusion. Bone density can be seen inside and between the cages. B: A lateral radiograph obtained 24 months postoperatively, demonstrating bone bridging between the vertebral endplates. C: Axial CT scan at L5-S1 demonstrating fusion bone between and beside the cages. The radiolucent HYDROSORB devices can be clearly demonstrated, although they are beginning to become amorphous. D: Coronal CT reconstruction at 24 months. E: Sagittal CT reconstruction at 24 months.

In the patient with a four-level fusion, there was a failure to fuse at one of the four levels. The probability of satisfactory fusion at all levels in a given patient decreased as the number of levels fused increased; this is consistent with previously published studies. ${ }^{4}$ Yet, when fusion success was analyzed according to the number of levels fused per patient, the fusion rate remains similar, between 93.3 and $100 \%$ in single- to three-level fusions.

Pseudarthrosis or fusion failure occurred at the caudal end of a three- and a four-level fusion. These failures became evident and led to repeated surgery at 19 and 20 months after the index fusion procedure. Fusion failure was manifested by a sudden clinical deterioration associated with a disc space collapse, with varying degrees of cavitation or lucency around the HYDROSORB devices (Figs. 4 and 5). At repeated operation there was no evidence of an inflammatory response but simply a fluidfilled void at the site of the HYDROSORB spacer. The remaining levels that appeared to be fused on radiographic studies were confirmed to be solid intraoperatively.

No significant surgical complication occurred; complications are listed in Table 3. Dural tears, permanent nerve root injury, infection, and death rates are recorded. There were four incidental dural tears recognized and repaired at the time of surgery; there were no postoperative cerebrospinal fluid leaks. No patient sustained a permanent nerve root injury. There were no infectious complications or deaths in any of the groups.

Standardized clinical outcome assessment was confounded by the fact that only 21 of the patients included in

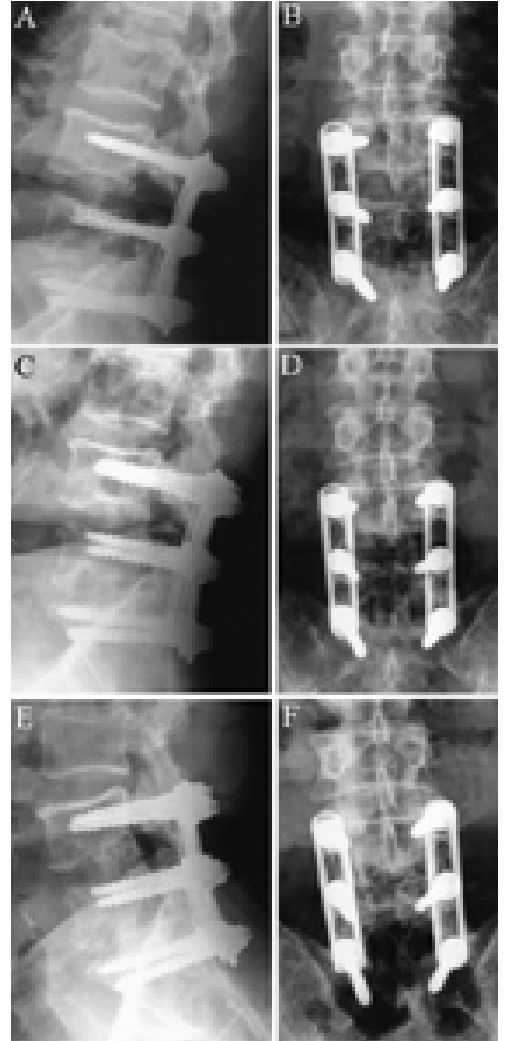

Fig. 2. Lateral (A, C, and $\mathrm{E})$ and $\mathrm{AP}(\mathrm{B}, \mathrm{D}$, and $\mathrm{F})$ radiographs obtained at different time periods after a two-level fusion with the HYDROSORB devices. A and B: Six-month follow up. C and D: Twelve-month follow up. E and F: Twenty-four-month follow up.

this 27-patient cohort elected to participate in a standardized outcome assessment algorithm. Of these 21, 16 patients completed 12-month and eight completed 24-month surveys, the results of which are shown in Fig. 6. All patients in the series reported a benefit derived from surgical treatment at the last follow-up review. It is difficult to confirm a statistically significant clinical benefit in this entire group by using these data, but that is not the intent of this report. No patient's condition was made worse.

\section{DISCUSSION}

The PLIF procedure accompanied by intervertebral spacers has an extensive history with a documented satisfactory fusion rate. . $^{1,4,6,9-11,15-19,21}$ We report the first 2-year follow-up study of a novel PLIF technique in which a biodegradable HYDROSORB interbody spacer was used.

Machine-prepared allograft bone in the form of wedges or threaded dowels has yielded a range of fusion results. ${ }^{3}$ Determining satisfactory on fusion radiographic studies may be difficult in the case of both cortical allograft and metallic devices. In addition, allograft bone may take many years to be incorporated or may never be completely replaced by host bone.

Although single-level instrumented PLIF with interbody cages or spacers is associated with a consistently 


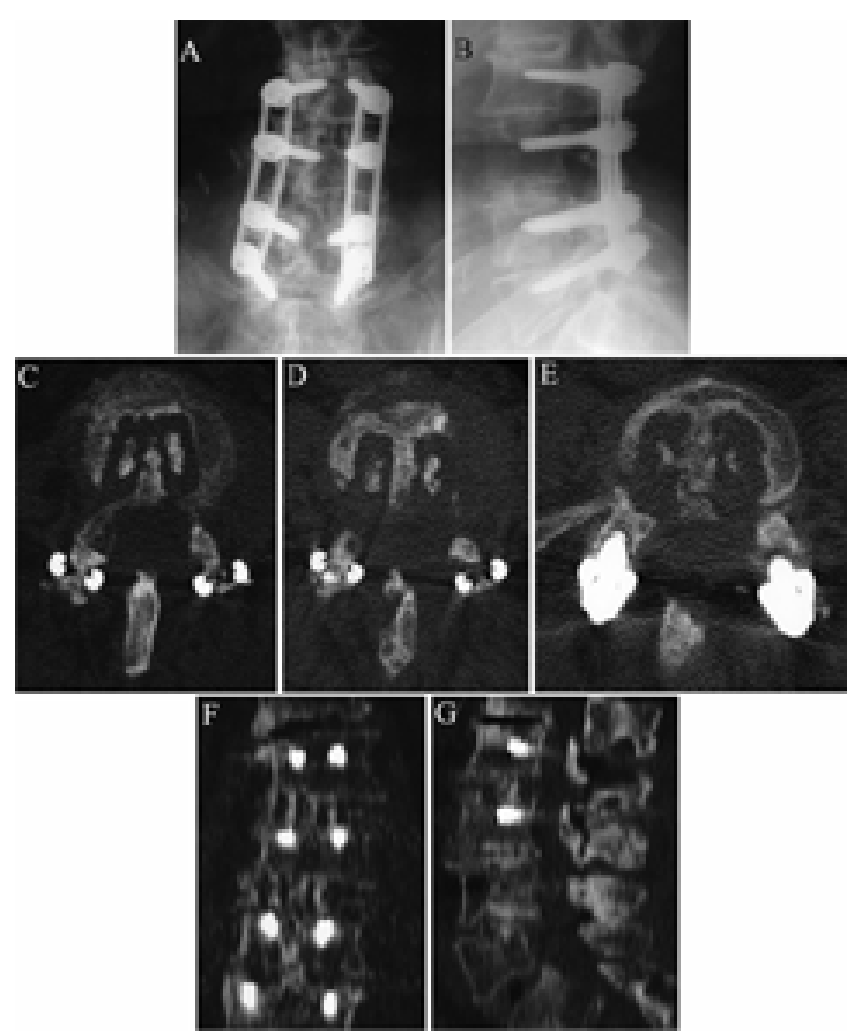

Fig. 3. A: An AP radiograph obtained 24 months after a threelevel fusion. Bone density can be seen inside and between the cages. B: A lateral radiograph obtained at 24 months, demonstrating bone bridging between the vertebral endplates at different levels. C-E: Axial CT scans demonstrating fusion bone between and beside the cages at the three different levels. The radiolucent HYDROSORB devices can be clearly demonstrated, and there is bone extending between and around the devices. F: Coronal CT reconstruction in the same patient. G: Sagittal CT reconstruction in the same patient. Adjacent-level disease can be seen above and below the construct.

high fusion rate, the type of cage may affect our ability to determine fusion status and it may potentially impact clinical outcome. Agazzi, et al., ${ }^{1}$ reported a $90 \%$ fusion rate after 28 months of follow up in 71 consecutive patients treated with metallic cages; the overall satisfaction rate was $67 \%$, with only $39 \%$ good or excellent results. Brantigan, et al. ${ }^{4}$ reported better results, with a fusion rate of $98.9 \%$ in 178 patients and a clinical success rate of $86 \%$. Carbon fiber or other synthetic cages are relatively radiolucent, yet the difficulties involved in the radiographic assessment of fusion with metallic intervertebral cages is an inherent limitation of many currently used devices. ${ }^{7,8,13,20}$

These limitations led to the development of biological spacers that provide immediate spinal stability, are radiolucent, and are ultimately biodegradable. The HYDROSORB biodegradable device allows more accurate assessment of fusion on conventional radiographic studies but imposes a time frame on the fusion process. In this early experience, the PLIF procedure with the HYDROSORB device appears to result in fusion rates that are comparable to those previously reported.

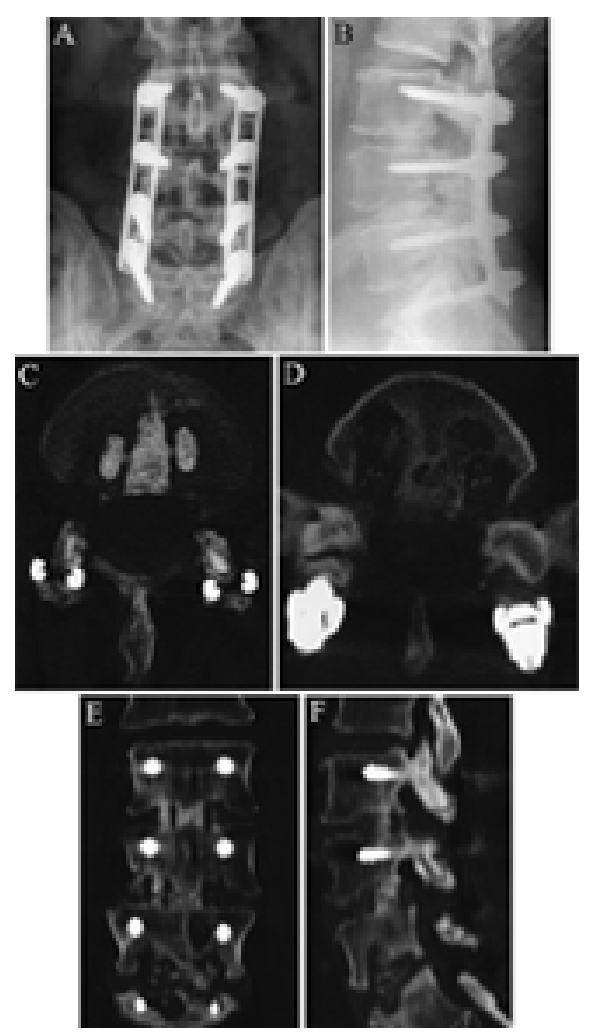

Fig. 4. Radiological studies obtained 18 months after a threelevel fusion. A pseudarthrosis developed at the L5-S1 level in this patient. A: An AP radiograph. B: A lateral radiograph. C: Axial CT view through the L3-4 level. D: Axial CT view through the L5-S1 level. Endplate erosion and cavitation of the vertebral body is noted. E: Coronal CT reconstruction with cavitation into the L-5 vertebral body. F: Sagittal CT reconstruction with solid fusion at L3-4 and L4-5 pseudarthrosis at L5-S1 and cavitation changes extending through the endplates at both L-5 and S-1.

Expected fusion levels in both the single- and multiplelevel fusion groups were attained. Clinical outcomes in this series appear to be consistent with those obtained using crushed cortical allograft bone preparations, although an extensive analysis of clinical outcome is outside the scope of this brief communication. The multiple-level pseudarthrosis rate was higher than in single-level fusions, as was found in previous studies, 4,5 in which a 25 to $30 \%$ pseudarthrosis in multiple-level fusion was reported. After 12 months, HYDROSORB devices begin to develop a consistent loss of strength due to resorption. If adequate fusion has not occurred within that time frame, there is increasing potential for disc space collapse with pseudarthrosis.

Analysis of data obtained in animal models indicates that at 12 months the HYDROSORB device retains 95\% of its structural integrity, yet in the face of the loads and motion caused by a subsequent fusion failure the biodegradation appears to increase, resulting in an appearance of cavitation comparable to the lucencies that appear around metallic or other devices in which a solid fusion has not been achieved. $7,8,13,20$ The exact mechanism or mechanisms associated with this somewhat striking cavitation appear- 

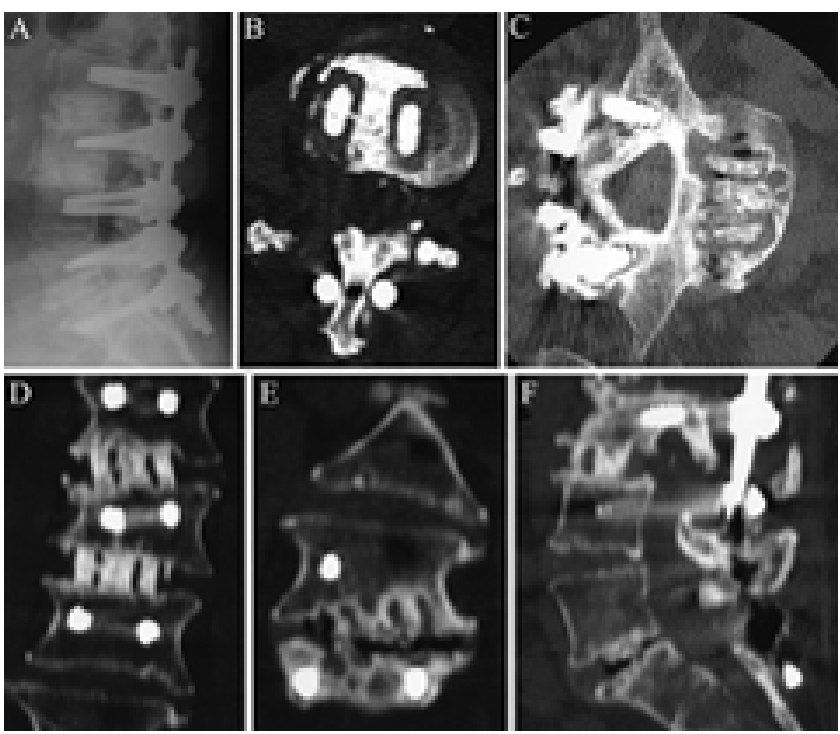

Fig. 5. Radiological studies obtained 18 months after a fourlevel fusion in a patient in whom a pseudarthrosis developed at L5-S1. HYDROSORB devices were placed at L2-3, L3-4, and L5-S1. A: A lateral radiograph. B: Axial CT scan of the L3-4 level demonstrating solid fusion. C: Axial CT scan of the L5-S1 level demonstrating pseudarthrosis. D: Coronal CT reconstruction demonstrating the L2-3 and L3-4 solid fusions. E: Coronal $\mathrm{CT}$ reconstruction demonstrating the L5-S1 pseudarthrosis. No cavitation changes were noted in this patient. F: Sagittal CT reconstruction illustrating the solid fusion at L3-4 and the pseudarthrosis at L5-S1 without the cavitation changes.
TABLE 3

Postoperative complications in 27 patients who underwent PLIF procedures

\begin{tabular}{lc}
\hline \hline \multicolumn{1}{c}{ Complication } & No. of Patients $(\%)$ \\
\hline incidental durotomy & $4(15)$ \\
permanent nerve root injury & $0(0)$ \\
infection (superficial or deep) & $0(0)$ \\
death & $0(0)$ \\
\hline
\end{tabular}

ance seen in conjunction with the bioabsorbable device certainly warrants further analysis.

Adequate interbody bone grafting in addition to implantation of the spacers optimizes the fusion process and appears to minimize the potential for fusion failure as the biodegradation of the device accelerates at 18 months. In our series, it appears that the fusion failures were consequences of inadequate interbody bone grafting or insufficient sacral fixation, especially at the extremes of a multiple-level fusion. These findings warrant further analysis of the efficacy of a bioabsorbable device in a patient with metabolic or structural characteristics that would retard the fusion process. We did recognize solid fusions in elderly women in whom multiple levels were fused.

In the patients who underwent single level fusions, two were found to have adjacent level disease during followup visits. Both of these patients underwent a single-level fusion at the adjacent level. A solid fusion was confirmed intraoperatively at the level previously fused. One of these

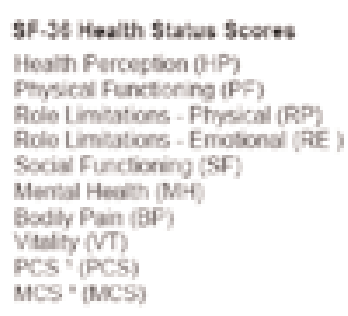

SF. 26 Mitan Scores

\begin{tabular}{|c|c|c|c|c|c|c|}
\hline Prosperative & Six Mo & inths & One & lear & Two Ye & ears \\
\hline 56.521 & 60.8 & 17 & 53.1 & 16 & 444 & $\gamma$ \\
\hline 20.422 & 435 & 17 & 414 & 17 & 30.4 & 8 \\
\hline 6822 & 255 & 17 & 219 & 16 & 36 & 7 \\
\hline 57.121 & 627 & 17 & 52.1 & 16 & 61.1 & 6 \\
\hline 3921 & 648 & 16 & E4 1 & 16 & 450 & b \\
\hline 63921 & 76.8 & 17 & 763 & 16 & 740 & 8 \\
\hline 22021 & 451 & 17 & 408 & 16 & 338 & 8 \\
\hline 29421 & 482 & 17 & 42.1 & 16 & $\$ 2.5$ & 8 \\
\hline 24321 & 313 & 15 & 30.1 & 15 & 234 & 5 \\
\hline 45.821 & 522 & 15 & 504 & 15 & 573 & 5 \\
\hline
\end{tabular}

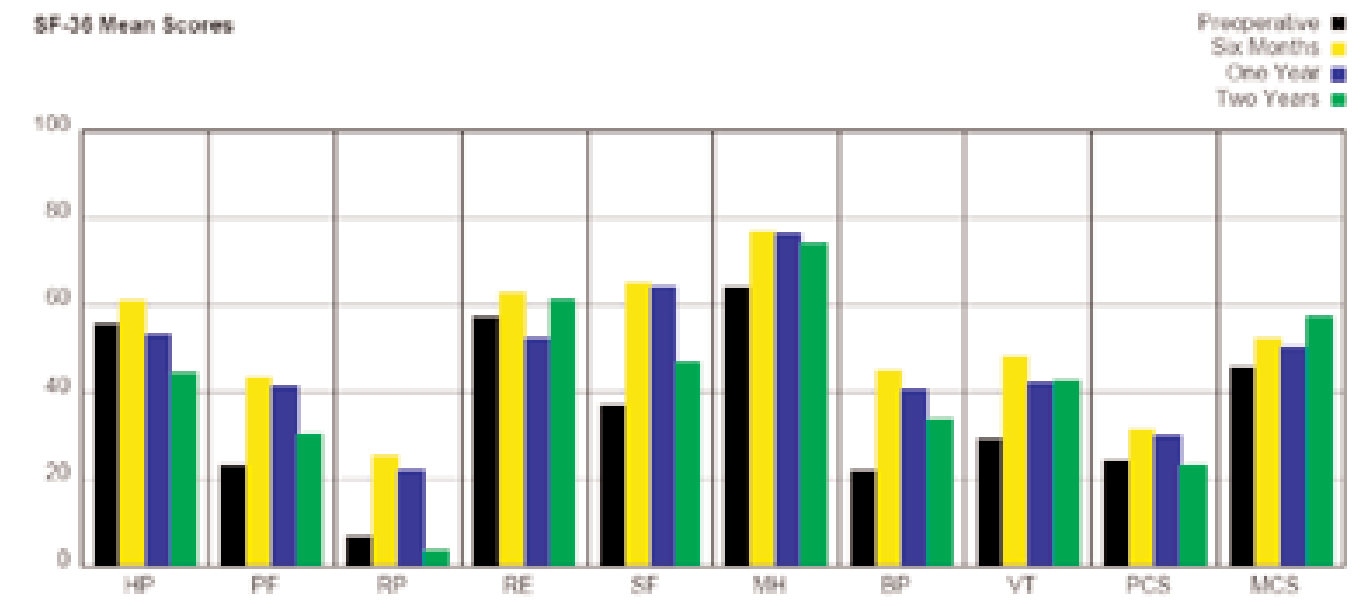

Fig. 6. Bar graphs showing the results of standardized clinical outcome assessment performed in 21 patients who underwent PLIF procedures. The second number in each column in the chart represents the number of patients who completed the follow-up evaluation at each interval. MCS = Mental Component Summary; PCS = Physical Component Summary; SF-36 = Short Form-36. 
patients went on to do well, whereas in the other a pseudarthrosis developed at the new level, which required a repeated operation; this patient's spinal column appeared to be fused at 12-month analysis.

We acknowledge that our study involves a small cohort documenting an early experience with a novel PLIF technique. This study does not include an outside independent review of the follow-up radiographs, yet the radiographic fusion rates documented here are consistent with previous reports and the integrity of the fusion was validated surgically in the cases in which a pseudarthrosis adjacent to a solid fusion was revised. We believe that the accuracy of our interpretation was facilitated by the radiolucent device used.

\section{CONCLUSIONS}

Our experience treating this small series of patients demonstrates that HYDROSORB bioabsorbable devices may be used to achieve satisfactory fusion rates and outcomes when used for PLIF procedures. The safety and efficacy of these devices appears to be comparable with other types of implants. We achieved expected fusion levels in single- and multiple-level groups. We recognize the time limitations imposed on the fusion process by a biodegradable support device; this warrants further analysis. The HYDROSORB implants appear to facilitate radiographic assessment of fusion, especially when compared with metallic devices.

\section{Disclosure}

Dr. Branch is a paid consultant with Medtronic Sofamor Danek.

\section{References}

1. Agazzi S, Reverdin A, May D: Posterior lumbar interbody fusion with cages: an independent review of 71 cases. J Neurosurg (Spine 2) 91:186-192, 1999

2. Alexander JT, Branch CL Jr, Subach BR, et al: Applications of a resorbable interbody spacer in posterior lumbar interbody fusion. J Neurosurg (Spine 4) 97:468-472, 2002

3. Barnes B, Rodts GE Jr, Haid RW Jr, et al: Allograft implants for posterior lumbar interbody fusion: results comparing cylindrical dowels and impacted wedges. Neurosurgery 51: 1191-1198, 2002

4. Brantigan JW, Steffee AD, Lewis ML, et al: Lumbar interbody fusion using the Brantigan I/F cage for posterior lumbar interbody fusion and the variable pedicle screw placement system: two-year results from a Food and Drug Administration investigational device exemption clinical trial. Spine 25:1437-1446, 2000
5. Brantigan JW: Pseudarthrosis rate after allograft posterior lumbar interbody fusion with pedicle screw and plate fixation. Spine 19:1271-1280, 1994

6. Brodke DS, Dick JC, Kunz DN, et al: Posterior lumbar interbody fusion. A biomechanical comparison, including a new threaded cage. Spine 22:26-31, 1997

7. Burkus JK, Foley KT, Haid RW, LeHuec JC: Surgical Interbody Research Group-radiographic assessment of interbody fusion devices: fusion criteria for anterior lumbar interbody surgery. Neurosurg Focus 10 (4):Article 11, 2001

8. Cizek GR, Boyd LM: Imaging pitfalls of interbody spinal implants. Spine 25:2633-2636, 2000

9. Elias WJ, Simmons NE, Kaptain GJ, et al: Complications of posterior lumbar interbody fusion when using a titanium threaded cage device. J Neurosurg (Spine 1) 93:45-52, 2000

10. Gill K, Blumenthal SL: Posterior lumbar interbody fusion: a 2-year follow-up for 238 patients. Acta Orthop Scand Suppl 251:108-110, 1993

11. Hutter CG: Posterior intervertebral body fusion. A 25-year study. Clin Orthop 179:86-96, 1983

12. Jaslow IA: Intercorporal bone graft in spinal fusion after disc removal. Surg Gynecol Obstet 82:215-222, 1946

13. Kant AP, Daum WJ, Dean SM, et al: Evaluation of lumbar spine fusion: Plain radiographs versus direct surgical exploration and observation. Spine 20:2313-2317, 1995

14. Klemme WR, Owens BD, Dhawan A, et al: Lumbar sagittal contour after posterior interbody fusion: threaded devices alone versus vertical cages plus posterior instrumentation. Spine 26: 534-537, 2001

15. Kuslich SD, Danielson G, Dowdle JD, et al: Four-year followup results of lumbar spine arthrodesis using the Bagby and Kuslich lumbar fusion cage. Spine 25:2656-2662, 2000

16. Kuslich SD, Ulstrom CL, Griffith SL, et al: The Bagby and Kuslich method of lumbar interbody fusion. History, techniques and 2-year follow-up results of a United States prospective, multicenter trial. Spine 23:1267-1279, 1998

17. Lin PM: Posterior lumbar interbody fusion technique: complications and pitfalls. Clin Orthop 193:90-102, 1985

18. Lund T, Oxland TR, Jost B, et al: Interbody cage stabilisation in the lumbar spine: biomechanical evaluation of cage design, posterior instrumentation and bone density. J Bone Joint Surg Br 80:351-359, 1998

19. Ray CD: Threaded titanium cages for lumbar interbody fusions. Spine 22:667-680, 1997

20. Siambanes D, Mather S: Comparison of plain radiographs and CT scans in instrumented posterior lumbar interbody fusion. Orthopedics 21:165-167, 1998

21. Zdeblick TA: A prospective, randomized study of lumbar fusion. Preliminary results. Spine 18:983-991, 1993

Manuscript received January 29, 2004.

Accepted in final form February 15, 2004.

Address reprint requests to: Charles L. Branch Jr., M.D., Professor and Chairman, Department of Neurosurgery, Wake Forest University Medical Center, Medical Center Boulevard, Winston-Salem, North Carolina 27105. email: cbranch@wfubmc.edu. 